\title{
Advanced dose calculation algorithm in superficial brachytherapy - the impact of tissue inhomogeneity on treatment plan dosimetry
}

\author{
Marta Szlag, PhD', Sylwia Kellas-Ślęczka, MD, PhD², Piotr Wojcieszek, MD, PhD², Magdalena Stankiewicz, MD, PhD², \\ Agnieszka Cholewka, MScl, Agnieszka Pruefer, MScl, Tomasz Krzysztofiak, MD², Piotr Lelek, MD², \\ Małgorzata Stapór-Fudzin’ska, PhD', Prof. Krzysztof Ślosarek, PhDl \\ ITreatment Planning Department, Maria Sklodowska-Curie National Research Institute of Oncology Gliwice Branch, Poland, \\ ${ }^{2}$ Brachytherapy Department, Maria Sklodowska-Curie National Research Institute of Oncology Gliwice Branch, Poland
}

\begin{abstract}
Purpose: Given tissue inhomogeneity and lack of backscatter media, superficial brachytherapy necessitates more accurate dosimetry than TG-43 formalism. However, the introduction of modern model-based dose calculation algorithms into clinical practice should be carefully evaluated. The aim of this work was to compare dose distributions calculated with TG-43 and advanced collapsed cone engine (ACE) algorithms for individual multi-catheter moulds, and investigate the impact of target size and the lack of bolus to differences between plans.

Material and methods: Eleven treatment plans for individual mould multi-catheter high-dose-rate brachytherapy (IMM HDR) were selected for retrospective analysis. All treatment plans were initially calculated with TG-43 formula and re-calculated using ACE algorithm. Plan re-calculation with ACE was repeated for each plan in order to assess the impact of bolus. To evaluate differences between TG-43 and ACE dose distributions, dose-volume histogram (DVH) parameters for each ROI were compared. $D_{\max }$ (maximal point dose), $\mathrm{D}_{0.1 \mathrm{cc}}$ and $\mathrm{D}_{2 \mathrm{cc}}$ were calculated for each risk's organ (OARs) and for external contour. For clinical target volume (CTV), $\mathrm{D}_{98}, \mathrm{D}_{90}, \mathrm{D}_{50}, \mathrm{CTV}$ coverage $\left(\mathrm{CTV}-\mathrm{V}_{100}\right)$, and dose delivered to reference point were compared between the plans.

Results: A significantly lower values $(p<0.05)$ of CTV parameters were observed for treatment plans calculated with ACE algorithm comparing to TG-43. Further analysis showed that differences between CTV-V ${ }_{100}$ for ACE and TG-43 plans depended on CTV volume. Dosimetric parameters for OARs were significantly lower in ACE plans than those of TG-43. Only $\mathrm{D}_{2 \mathrm{cc}}$ for external and $\mathrm{D}_{01 \mathrm{cc}}$ for both eye lenses in ACE plans were insignificantly different comparing to TG-43 plans.

Conclusions: Results show that differences between dosimetric parameters are statistically significant. However, their clinical relevance is still undetermined. Careful re-evaluation of the clinical results based on long-term research on TG-43 is necessary to safely introduce modern algorithms to clinical practice.

J Contemp Brachytherapy 2021; 13, 4: 441-446 DOI: https://doi.org/10.5114/jcb.2021.106541
\end{abstract}

Key words: skin brachytherapy, moulds, TG-186, model-based dose-calculation algorithms.

\section{Purpose}

Brachytherapy is widely known as an appropriate and effective method for non-melanoma skin cancers' (NMSC) treatment $[1,2]$. Currently, skin brachytherapy can be applied superficially (contact brachytherapy or plesiotherapy), or interstitially, with elastic catheters or rigid needles inserted to tumor mass $[1,3]$. Applicators used to deliver the dose to the skin are commercially available, such as Valencia, Leipzig, HAM, Freiburg flap, etc. or are individually prepared, based on patient's anatomy. Custom moulds or flaps are dedicated for various tumors size, while for smaller lesions, radionuclide-based shielded applicators and electronic-based shielded applicators are used $[1,2]$.
Moulds are designed to deliver a conformal dose distribution in a reproducible manner, especially in problematic planning areas, including curved area, head and neck region, lesions located close to crucial organs at risk, such as eye lens [1]. Individual mould multi-catheter high-dose-rate (IMM HDR) technique allows for precise adaptation of the dose to a treated region [3]. In our institute, moulds have been widely used for NMSC. We adapted dental materials and dental imprints manufacturing to prepare the imprints of patient anatomy. Treatment planning and dose calculation methods for IMM HDR evolved with time and changed significantly when 3D imaging modalities entered in routine practice. While treatment planning has evolved, taking an advan- 
tage from anatomical information delivered by computed tomography (CT), final dose distribution calculation suffered the lack of precision due to limitations embedded in dose calculation algorithm, which is routinely applied in brachytherapy planning systems. Dose calculations provided by TG-43 algorithm are based on single-source in homogenous all-water environment [4]. Nowadays, technological advancement enabled more accurate dose calculation using model-based dose calculation algorithms (MBDCAs) presented in the AAPM TG-186 report in 2012 [4]. These algorithms provide more accurate computation of actual dose delivered to patient.

MBDCAs, implemented in Oncentra Brachy planning system (Elekta AB, Sweden), exploits kernel superposition/convolution method. Collapsed cone convolution (CCC), along with a commercial advanced collapsed cone engine (ACE) algorithm can potentially result in a more accurate computation of actual dose delivered to patient and significantly improve precision of brachytherapy treatment.

Superficial brachytherapy is an example of treatment, in which introduction of advanced algorithms (apart of the TG-43 formalism) seems to be desirable and beneficial, especially for the head and neck region, where tissue inhomogeneity in a short distance from the source may pose limitations to the TG-43 dosimetry. The impact of complex tissue composition (other than all-water TG-43 environment) and the lack of scattering medium above the radioactive source should be carefully evaluated with a new algorithm before its clinical use $[4,5]$.

For treating NMSC, a wide range of techniques and prescription methods are successfully used across all radiotherapy centers [1]. Each technique and tumor location are generally expected to show different level of dosimetric susceptibility when changing to MBDCAs. In our department, superficial moulds are commonly used to treat tumors of different size and locations. Due to insufficient number of current published papers, little is known about the scale of clinical impact related to the use of MBDCAs for this specific brachytherapy technique. For these reasons and to comply with professional society recommendations, we decided to compare the dose distributions generated with the TG-43 and ACE algorithms for superficial, individual multi-catheter moulds. Additionally, we investigated to what extend the target size and the lack of bolus material can influence and modify the differences between plans calculated with these two algorithms.

\section{Material and methods}

Individual mould multi-catheter surface HDR applicator is a plate that is made of dental thermoforming foil used for the production of occlusal splints. The material density is $1.27 \mathrm{~g} / \mathrm{cm}^{3}$. The plate is a model of individual patient's anatomy, and is formed based on the plaster sculpture. In our study, the thickness of applicators' plate for nine patients was $3 \mathrm{~mm}$ (thin plate), and two patients had $6 \mathrm{~mm}$ plate applicator (thick plate). Thicker plates were prepared intentionally to increase the specification depth to $10 \mathrm{~mm}$. In order to produce an applicator, a two- phase silicon mass was used for negative imprint of the patient skin in the region of the tumor. The imprint was a mould for moistened plaster. Finally, a plate was extruded on the plaster sculpture.

$6 \mathrm{~F}$ plastic catheters (Elekta $\mathrm{AB}$, Sweden) were fixed to the outer surface of plate. The number and configuration of the catheters depended on the size, shape, and location of clinical target volume (CTV). Number of catheters increased for curved surfaces and large tumors. For flat surfaces, the catheters were usually equidistant and parallel. The distance between catheters on average was $8 \mathrm{~mm}$ (range, 6-10 mm). For curved surfaces (tip of the nose, ear, and eye corner), the number of catheters may raise and the distance may vary significantly.

According to the GEC-ESTRO ACROP recommendations, CTV was defined as a visible tumor mass, with 5-10 mm margin (depending on the clinical situation). The CTV thickness ranged from $2 \mathrm{~mm}$ to $5 \mathrm{~mm}$. CTV was marked on patient's skin and copied on an applicator's plate.

Eleven treatment plans for patients with basal cell carcinoma located on the nose, treated with individual mould multi-catheter HDR brachytherapy (iridium-192) were randomly selected for retrospective dosimetric analysis. Although, in our department, we treat about 200-300 patients with NMSC per year, and 90\% of them are treated with individual moulds, only 11 patients treated in 2019 were qualified for the present study. These patients were examined with the same CT scanner, installed in our brachytherapy department in 2018. Applicators prepared for these patients were also produced by the same specialist. In order to minimize the impact of collateral parameters, which may impede a conclusion-making process, we followed restrictive criteria when selecting patients for analysis. All patients were treated with individual moulds of similar size, geometry, and curvature. Moreover, similar location of the lesion was preferable. In order to homogenize the analysis group as far as possible, only tumors located on the nose were included for analysis. The group of patients consisted of the most recent patients treated in our department because of the learning curve process. We needed to assure that plans have been prepared based on the same rules and actual knowledge.

Lesions were located on the nasal bridge, nasal sidewall, and ala of the nose. In this anatomical region, tissue composition is complex and inhomogeneous due to the presence of media represented by atomic numbers and mass densities related to air, soft tissues, and bones. All analyzed treatment plans were CT-based. Discovery RT (GE Healthcare) computed tomography was used for scanning, with slice thickness of $1.25 \mathrm{~mm}$. Patient's CT scanning was performed with the applicator in place. CTV and organs at risks (OARs) were contoured on CT scans. Applicator plate was also contoured and assigned as a brachytherapy device in a region of interest (ROI) contour set. External patient body was contoured automatically, and corrected manually to eliminate the inaccuracy of automatic volume recognition algorithm. Low density natural air cavities (sinuses) were taken into account while contouring the external and subtracted from the external structure. ROI set that was contoured for treatment planning and plan optimization included the 
skin surface (external contour), bone, eye globes, and eye lenses. No backscatter bolus was used during CT scanning and treatment.

Dose specification point (reference point) was located 2-5 mm below the skin (on the bottom surface of CTV). For two patients, the depth of reference point was $10 \mathrm{~mm}$. Specification depth varied depending on skin thickness and visibility of the tumor mass. The patients' plans were done using Oncentra Brachy planning system (Elekta AB, Sweden) v. 4.6.0, according to the internal institutional protocol, which assumes delivering a total dose of $45 \mathrm{~Gy}$ in 9 fractions to the reference point. Treatment was delivered with MicroSelectron v. 3 HDR (Elekta AB, Sweden). Reference dose should cover at least $90 \%$ of CTV. Moreover, maximal point dose delivered to the skin (external contour) must not exceed $200 \%$ of prescribed dose, and maximal dose to the bone structures should be lower than $100 \%$ of reference dose. Therefore, maximal doses to the eye lenses were reported in each plan and limited if possible. During the treatment, lead plates were used to protect the eyes (lead shielding was not considered during treatment planning and dose distribution calculation with ACE algorithm).

All treatment plans were initially calculated with the TG-43 (TG-43 plans) and recalculated using the ACE algorithm (ACE plans). The TG-43 as well as ACE plans were calculated in the same treatment planning system (Oncentra Brachy), using the same planning station. The same calculations grid was used for each parallel computation. Dwell times and number of dwell positions in each catheter remained unchanged for each re-calculation process. In contrast to the TG- 43 formalism, the model-based approach required treatment applicator and patient's anatomy to be adequately described before dose computation was proceeded. For each ROI, material type and calculation Hounsfield unit (HU)-based method was assigned. Applicator plate was ascribed as a brachytherapy device made of polyphenylsulfone (PPSU), with a density of $1.29 \mathrm{~g} / \mathrm{cm}^{3}$.

Treatment plans re-calculation with the ACE algorithm was repeated for each plan in order to assess the impact of additional backscatter material introduced to the treatment plan (ACE-bolus plans) (Figures 1 and 2). This structure simulated a bolus filled with water and placed directly on the applicator. CTV of $\mathrm{V}_{100}$ was cal-



Fig. 1.3D reconstruction of external contour of patient and applicator plate with catheters for TG-43 and advanced collapsed cone engine (ACE) treatment plans calculation culated for ACE-bolus plans and compared with values obtained for ACE and TG-43 plans.

To evaluate differences between dose distributions computed with the TG-43 and ACE algorithms, the group of clinically relevant dose-volume histogram (DVH) parameters were selected for each ROI. $D_{\max }$ (maximal point dose), $\mathrm{D}_{01 \mathrm{cc}}$ and $\mathrm{D}_{2 \mathrm{cc}}$ were calculated for each organ at risk and for external contour. $\mathrm{D}_{98}, \mathrm{D}_{90}$, and $\mathrm{D}_{50}$ parameters, which stand for dose delivered to $98 \%, 90 \%$, and $50 \%$ of CTV, respectively, were calculated from cumulative DVH. CTV coverage $\left(\mathrm{CTV}-\mathrm{V}_{100}\right)$ was also evaluated and compared between plans as well as dose delivered to the reference point.

Sample size used for this experiment was relatively small. Small sample size could decrease the power of statistics and for such data, non-parametric tests would be recommended. In our study, statistical significance between the groups was estimated with dependent $t$-Student test (for normally distributed data) or Wilcoxon signed-rank test. For $t$-test, the power of statistical test was conducted to justify the sample size. The power of the test was above 0.85 level. A threshold value for significance level ( $p$-value) was set to 0.05 . Mean values were presented with one standard deviation. Shapiro-Wilk test was used to determine whether the data sets had a normal distribution.

\section{Results}

\section{Dose to CTV}

Statistically significant difference was observed when comparing the dosimetric and volumetric parameters of dose distributions calculated with the TG-43 and ACE algorithms. A significantly lower values $(p<0.05)$ of CTV parameters $\left(\mathrm{CTV}-\mathrm{V}_{100}, \mathrm{D}_{98}, \mathrm{D}_{90}\right.$, and $\left.\mathrm{D}_{50}\right)$ were observed for treatment plans calculated with the ACE algorithm in comparison to the TG-43 formula (Table 1). The TG-43 overestimated both target coverage and dose delivered to the reference point. The mean total dose in the reference point was reduced to $43.2 \pm 0.6 \mathrm{~Gy}$ for the ACE plans as compared with 45 Gy for the TG-43 plans. Analysis revealed $10.7 \pm 6.5 \%$ mean difference in target coverage $\left(\mathrm{CTV}-\mathrm{V}_{100}\right)$ between the ACE and TG-43 treatment plans. CTV $-V_{100}$ below $90 \%$ was observed in $64 \%(7 / 11)$ of the

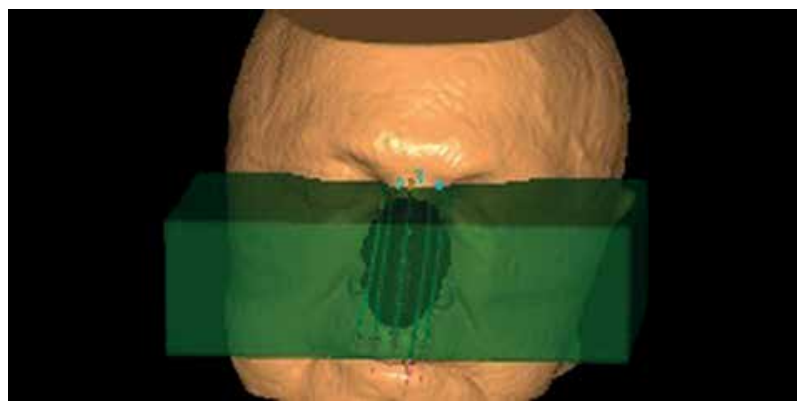

Fig. 2. 3D reconstruction of contours for advanced collapsed cone engine (ACE)-bolus plans calculations. Additional bolus structure (green, semi-transparent block) was added to ROI set 
Table 1. Summary of median and mean values (with one standard deviation) of dosimetric parameters for clinical target volume (CTVs) calculated with advanced collapsed cone engine (ACE) and TG-43 algorithms

\begin{tabular}{lccccc} 
& \multicolumn{3}{c}{ TG-43 } & \multicolumn{2}{c}{ ACE } \\
\cline { 2 - 6 } & Median & Mean (SD) & Median & Mean (SD) & $p$-value \\
\hline CTV-V $_{100}(\%)$ & 95.9 & $94.7(5.0)$ & 84.3 & $84.1(8.6)$ & 0.003 \\
\hline $\mathrm{D}_{98}(\%)$ & 97.8 & $98.5(6.7)$ & 94.0 & $93.3(5.2)$ & 0.003 \\
\hline $\mathrm{D}_{90}(\%)$ & 102.7 & $103.1(4.9)$ & 98.0 & $98.8(5.0)$ & 0.003 \\
\hline $\mathrm{D}_{50}(\%)$ & 114.7 & $117.8(8.3)$ & 109.8 & $113.1(8.7)$ & 0.003
\end{tabular}

$D_{50}, D_{90}, D_{98}$ - relative dose delivered to $50 \%, 90 \%, 98 \%$ of the target (\%), CTV-V $V_{100}$ - relative volume of the CTV structure receiving $100 \%$ of the prescribed dose, p-values for Wilcoxon signed-rank test statistic were used to estimate the statistical significance of differences between medians in the two groups



Fig. 3. Dependence of relative difference in clinical target volume (CTV) coverage (CTV-V $\mathrm{V}_{100}$ (TG-43-ACE)) on CTV volume for two applicator thickness of $3 \mathrm{~mm}$ and $6 \mathrm{~mm}$

analyzed plans (re-calculated with the ACE algorithm). The mean CTV-V $\mathrm{V}_{100}$ for the ACE plans with artificial water bolus (ACE-bolus plans) was also lower, but the mean difference was only $3.2 \pm 2.2 \%$.

Further analysis revealed that differences between CTV-V $\mathrm{V}_{100}$ for the ACE and TG-43 plans depended on CTV volume. Differences between target coverage $\mathrm{CTV}-\mathrm{V}_{100}$ (TG-43-ACE) increased proportionally with the increase in CTV volume (Figure 3). The slope calculated for the patient with a standard size applicator (thin plate) was 4.6. Differences in CTV coverage between the TG- 43 and ACE plans for two patients treated with $6 \mathrm{~mm}$ plate were relatively large, and exceeded $18 \%$. However, the observed differences were lower than anticipated, based on the extrapolated data for the group of patients with $3 \mathrm{~mm}$ applicators (Figure 3). Dose specification depth and CTV volume for two patients treated with $6 \mathrm{~mm}$ plate were larger than in the thin plate group. Smaller than expected differences observed for the thicker applicators could occur from greater specification depth, in which the dose profile was less steep or with more homogenous tissue composition.

\section{Dose to OARs}

Median and mean values with one standard deviations of dosimetric parameters $\left(D_{\max }, D_{0.1 c c}\right.$ and $\left.D_{2 c c}\right)$ for organs at risk are presented in Table 2. Mean values of maximal point dose, $\mathrm{D}_{0.1 \mathrm{cc}}$ and $\mathrm{D}_{2 \mathrm{cc}}$ for external contour in the ACE plans were significantly lower than those observed in the TG-43 group $(p<0.05)$. Dosimetric parameters $\left(\mathrm{D}_{\max }, \mathrm{D}_{0.1 \mathrm{cc}}\right.$, and $\left.\mathrm{D}_{2 \mathrm{cc}}\right)$ calculated for the bone

Table 2. Summary of median and mean relative values with (with one standard deviation) of dosimetric parameters for organs at risks (OARs) calculated with advanced collapsed cone engine (ACE) and TG-43 algorithms

\begin{tabular}{|c|c|c|c|c|c|c|}
\hline & & \multicolumn{2}{|c|}{ TG-43 } & \multicolumn{2}{|c|}{ ACE } & \multirow[t]{2}{*}{$p$-value $e^{* * *}$} \\
\hline & & Median & Mean (SD) & Median & Mean (SD) & \\
\hline \multirow[t]{3}{*}{ External } & $\mathrm{D}_{\max }(\%)$ & 181.4 & $183.1(11.1)$ & 169.3 & $169.8(11.5)$ & $<0.001(\mathrm{t})$ \\
\hline & $\mathrm{D}_{0.1 \mathrm{cc}}(\%)$ & 149.2 & $149.9(7.1)$ & 142.0 & $144.0(8.9)$ & $0.007(\mathrm{t})$ \\
\hline & $\mathrm{D}_{2 \mathrm{cc}}(\%)$ & 104.1 & $107.2(11.5)$ & 100.4 & $102.5(10.3)$ & $<0.001(\mathrm{t})$ \\
\hline \multirow[t]{3}{*}{ Bone } & $\mathrm{D}_{\max }(\%)$ & 99.4 & $96.3(15.1)$ & 93.7 & $91.1(14.9)$ & $0.003(\mathrm{~W})$ \\
\hline & $\mathrm{D}_{0.1 \mathrm{cc}}(\%)$ & 91.8 & 83.5 (19.3) & 85.8 & 77.7 (18.3) & $0.003(\mathrm{~W})$ \\
\hline & $\mathrm{D}_{2 \mathrm{cc}}(\%)$ & 40.0 & 38.8 (15.9) & 36.9 & 35.8 (14.9) & $<0.001(\mathrm{t})$ \\
\hline \multirow{3}{*}{$\begin{array}{l}\text { Eye lenses } \\
\text { (left and right) }\end{array}$} & $\mathrm{D}_{\max }(\%)$ & 13.25 & $15.3(7.9)$ & 13.2 & $15.0(7.7)$ & $0.03(W)$ \\
\hline & $\mathrm{D}_{0.1 \mathrm{cc}}(\%)^{\star *}$ & 11.2 & $12.5(6.4)$ & 11.05 & $12.6(6.7)$ & $0.13(\mathrm{~W})$ \\
\hline & $D_{2 c c}(\%)^{*}$ & - & - & - & - & - \\
\hline
\end{tabular}

$D_{\text {max }}, D_{0.1 c c}$ and $D_{2 c c}$ dose delivered to maximal dose point, $0.1 \mathrm{cc}$ and $2 c c$ of the organ at risk, ${ }^{*} D_{2 c c}$ was not available for eye lens. The organ's volume was smaller than $2 \mathrm{cc},{ }^{* *}$ Dosimetric parameter calculated with ACE and TG-43 not statistically different, ${ }^{* * *}(W)-p$-values for Wilcoxon signed-rank test were used to estimate the statistical significance of differences between medians in the two groups, ${ }^{* * *}(t)-p$-values for $t$-Student test was used to estimate the statistical significance of differences between mean values in the two groups 
structure and maximal point doses delivered to the lens (both right and left) were also significantly lower in the ACE plans. Only $\mathrm{D}_{0.1 \mathrm{cc}}$ for both lenses in the ACE plans differed insignificantly as compared to the TG-43 plans. Dose delivered to $0.1 \mathrm{cc}$ of lens was almost equal in both the TG-43 and ACE treatment plans.

\section{Discussion}

Dose calculation in brachytherapy is based mainly on TG-43 approach. This widely adopted in modern treatment planning systems protocol provides dose distribution, which is valid only for homogeneous water phantom with infinite dimensions [4, 6]. Calculation of 3D dose distribution with this formalism is fast and efficient in clinical practice, but suffers certain inherent and formidable drawbacks, while tissue and applicator inhomogeneities are ignored [4].

Several recently developed MBDCAs are capable of modelling radiation transport in heterogeneous media [6], resulting in a more accurate dose distribution actually delivered to patient [4]. However, treatment planning system verification protocol necessitate an introduction of reliable and repeatable methods before clinical use of the MBDCAs algorithm. Monte Carlo simulations and film dosimetry performed in many research papers validated the results obtained with MBDCAs algorithm [5, 7] Zwierzchowski et al. proposed a verification procedure based on self-developing dosimetry films, which could be successfully used for treatment planning system verification before the transition to MBDCAs [8].

In our study, the ACE algorithm was used to re-calculate 11 superficial brachytherapy plans for skin tumors located on the nose, initially computed with the TG-43 protocol. Despite the small sample size, the power analysis of statistical test confirmed that the results are adequate and lead to reliable conclusions.

The results of this study indicate that the lack of backscatter and tissue heterogeneity correction significantly influenced dosimetric parameters of the treatment plans for this tumor location. Presented results showed that differences in target coverage between treatment plans may exceed $18 \%$ and increase with the growth of CTV volume. However, similar analysis performed recently in several studies demonstrated that the impact of heterogeneity corrected dose calculation on intracavitary treatment or interstitial breast brachytherapy was less pronounced [9-11]. Jacob et al. reported that for unshielded plastic gynecologic applicators, only minor dosimetric changes were found using MBDCAs in relation to standard TG-43 [9]. Hofbauer et al. retrospectively evaluated dose heterogeneity for cervix cancer patients. Doses to OARs were reduced up to $2 \%$ per fraction, while for high-risk CTV, they were reduced up to $0.5 \%$ per fraction [11]. Similarly, the impact of heterogeneity corrected dose calculation using a grid-based Boltzmann solver on breast was investigated in clinical treatment planning and phantom studies. However, the observed results for breast were not considered clinically relevant by the authors [11]. Mikell et al. also used grid-based Boltzmann equation solver (GBBS) Acuros to investigate dosimetric impact of het- erogeneity dose calculation for cervical cancer patients brachytherapy. The results of this survey also revealed that the impact on clinical parameters for patients treated with unshielded applicators is minimal [10].

Although the medium inhomogeneity might appear to affect only superficial brachytherapy, Vijande et al. suggested that overall impact of applicator composition and shape with the surrounding air on a clinical superficial plan was minor. They concluded that TG- 43 formalism could be used for a standard applicator within the clinical tolerance level [12]. Our findings do not support these observations. In the present study, CTV coverage differences exceeded $5 \%$ in $72 \%$ of the analyzed plans. In $64 \%$ of the plans, acceptance criteria were not met after dose re-calculation with the ACE algorithm. Only the mean relative difference in the total dose delivered to the reference point was less than $5 \%$.

In a study, Guinot et al. suggested that no bolus over a mould was required because differences at the prescription depth between TG-43 and Monte Carlo calculations were insignificant for ${ }^{192} \operatorname{Ir}[1,13]$. In our study, the use of bolus above the applicator located on the nose reduced considerably the difference between the TG- 43 and ACE plans. The applicator design (plate thickness and its curvature) as well as treated anatomy may potentially moderate the dosimetric response to the algorithm used for plan calculation. We assume that complex medium composition of the anatomical region, such as nose, may have a significant impact on dose calculation accuracy. In our study, we observed that the use of a bolus reduced the difference between dose distribution obtained with the TG-43 and ACE formalisms.

Boman et al. observed that differences between plans calculated with two formalisms resulted also from differences in loading areas. Deviations between TG-43 and Acuros dose distributions provided in the absence of backscatter material increased with the growth of loading area [5]. This is in an agreement with our findings, where the $C T V-V_{100}$ for ACE decreased with the increase of CTV volume.

To a certain extent, dose that is delivered to the patient correlates with clinical outcomes. The obtained results demonstrated that the delivered dose could differ from the calculated dose, and the differences between dosimetric parameters obtained with the two formalisms were statistically significant. However, their clinical relevance is still unknown since statistical significance cannot be transposed directly into clinical use.

In the present study, dose prescription was based on the TG-43 algorithm according to the TG-186 Working Group recommendations. However, our analysis indicated that the delivered dose was lower than the prescribed dose but this lower dose was in associated with long-term clinical outcomes. Transition to the ACE algorithm while keeping the same prescription method and dose value would increase the dose delivered to the patient. This could potentially start a new data collecting process in order to reveal a correlation between dose and clinical outcome, despite the fact that dose reporting would remain the same.

Changing the dose prescription in superficial brachytherapy by implementing model-based dose calculation 
algorithm may improve the consistency of calculated and delivered dose but, at the same time, it affect clinical treatment outcomes.

\section{Conclusions}

In our study, we evaluated and compared the dosimetric parameters of the treatment plans calculated with the TG-43 and ACE algorithms for superficial, individual multi-catheter moulds. The obtained results showed that differences between the dosimetric parameters selected for analysis were statistically significant.

Dose delivered to the target structure and organs at risk were overestimated when all-water algorithm was used for calculations. Further analysis revealed that the differences between CTV coverage for the ACE and TG-43 plans depended on the CTV volume, and proportionally increased with the growth of CTV volume.

Implementation of the dose calculation algorithms of higher accuracy in superficial brachytherapy is therefore desirable. However, possible future modifications of dose prescribing method should be considered between physicist and radiation oncologist specialists, and after careful re-evaluation of clinical results based on long-term research and experience obtained with TG-43.

\section{Disclosure}

The authors report no conflict of interest.

\section{References}

1. Guinot JL, Rembielak A, Perez-Calatayud J et al. GECESTRO ACROP recommendations in skin brachytherapy. Radiother Oncol 2018; 126: 377-385.

2. Delishaj D, Rembielak A, Manfredi B et al. Non-melanoma skin cancer treated with high-dose-rate brachytherapy: a review of literature. J Contemp Brachytherapy 2016; 8: 533-540.

3. Szlag M, Wojcieszek P, Kellas-Ślęczka $S$ et al. Individual multi-catheter mould technique in high- dose-rate brachytherapy - personalized approach in treating multifocal angiosarcoma of the face. J Contemp Brachytherapy 2019; 11: 337-342.

4. Beaulieu L, Tedgren AC, Carrier JF et al. Report of the Task Group 186 on model-based dose calculation methods in brachytherapy beyond the TG-43 formalism: Current status and recommendations for clinical implementation. Med Phys 2012; 39: 6208-6236.

5. Boman E, Satherley T, Schleich $\mathrm{N}$ et al. The validity of Acuros BV and TG-43 for high-dose-rate brachytherapy superficial mold treatments. Brachytherapy 2017; 16: 1280-1288.

6. Fonseca GP, Tedgren AC, Reniers B et al. Dose specification for 192Ir high dose rate brachytherapy in terms of dose-towater-in-medium and dose-to-medium-in-medium. Phys Med Biol 2015; 60: 4565-7459.

7. Ma Y, Vijande J, Ballester $F$ et al. A generic TG-186 shielded applicator for commissioning model-based dose calculation algorithms for high-dose-rate 192Ir brachytherapy Med Phys 2017; 44: 5961-5976.

8. Zwierzchowski G, Bielęda G, Skowronek J et al. Film based verification of calculation algorithms used for brachytherapy planning-getting ready for upcoming challenges of MBDCA. J Contemp Brachytherapy 2016; 8: 326-335.

9. Jacob D, Lamberto M, DeSouza Lawrence L et al. Clinical transition to model-based dose calculation algorithm: a retrospective analysis of high-dose-rate tandem and ring brachytherapy of the cervix. Brachytherapy 2017; 16: 624-629.

10. Mikell J, Klopp A, Gonzalez G et al. Impact of heterogeneity-based dose calculation using a deterministic grid-based boltzmann equation solver for intracavitary brachytherapy. Int J Radiat Oncol Biol Phys 2012; 83: 417-422.

11. Hofbauer J, Kirisits C, Resch A et al. Impact of heterogeneity-corrected dose calculation using a grid-based Boltzmann solver on breast and cervix cancer brachytherapy. J Contemp Brachytherapy 2016; 8: 143-149.

12. Vijande J, Ballester F, Ouhib F et al. Dosimetry comparisons between TG-43 and Monte Carlo calculations using the Freiburg flap for skin high-dose-rate brachytherapy. Brachytherapy 2012; 11: 528-535.

13. Granero D, Perez-Calatayud J, Vijande J et al. Limitations of the TG-43 formalism for skin high-dose-rate brachytherapy dose calculations. Med Phys 2014; 41: 021703. 